Im Rahmen des auf Bachelorstufe angesiedelten Major Clinical Medicine bot die Medizinische Fakultät der Universität Basel den Medizinstudierenden des 3. Jahreskurses in diesem Jahr das Interprofessionelle Modul «Ich werde Zeitschriftenmacher(in)! Jobs im medizinischen Fachverlag» an, das in Zusammenarbeit mit dem Schweizerischen Ärzteverlag EMH realisiert wurde. Sieben Medizinstudentinnen und drei Medizinstudenten waren im Zeitraum von März bis Mai an fünf Halbtagen bei EMH zu Gast, um den Redaktions- und
Produktionsprozess medizinischer Fachzeitschriften kennenzulernen. Der Kurs sollte eine enge Verbindung von Theorie und Praxis bieten - die Studierenden hatten sich auch als Journalisten und Redaktorinnen zu bewähren. Nachfolgend präsentieren Denise Bernhard, Marionna Cathomas, Rebekka Eugster, Mirjam Fröhlich und Claudia Schmid die Früchte ihrer Arbeit.

Dr. med. et lic. phil. Bruno Kesseli, Chefredaktor

\title{
Die zwei Gesichter des Bologna-Systems
}

\author{
Nachdem im Juni 1999 in Bologna die gemeinschaftliche Erklärung der europäi- \\ schen Bildungsminister zur Einführung eines gemeinsamen Hochschulraumes \\ eingereicht wurde, sind wir Medizinstudenten nun auch von dieser Reform be- \\ troffen. Die Gegenüberstellung von gemeinschaftlichem Interesse der Öffentlich- \\ keit und dem persönlichen Interesse eines einzelnen Studenten führt zu einer dif- \\ ferenzierten Wahrnehmung von «Bologna».
}

\section{Denise Bernhard,}

Rebekka Eugster
1 Gemeinsame Erklärung der Europäischen Bildungsminister vom 19. Juni 1999 , Bologna. Deutsche Fassung im Wortlaut nachzulesen z. B. unter http://www.bmbf.de/ pub/bologna_deu.pdf

\footnotetext{
* European Credit Transfer
} and Accumulation System

\section{Korrespondenz:}

zeitschriftenmacher@ hotmail.com

\section{Das Bologna-System}

Idee des Bologna-Systems ist es, die internationale Wettbewerbsfähigkeit der Studienabgänger auf dem Arbeitsmarkt zu verbessern und die Abschlüsse leichter verständlich und vergleichbarer zu machen. Als Instrument für grösstmögliche Mobilität wurde ein Leistungspunktesystem eingeführt. Den Teilnehmern der Konferenz war es ein wichtiges Anliegen, ein «wachsendes Bewusstsein für die Notwendigkeit der Errichtung eines vollständigeren und umfassenderen Europa [zu schaffen], wobei wir insbesondere auf seinen geistigen, kulturellen, sozialen und wissenschaftlich-technologischen Dimensionen aufbauen und diese stärken sollten.» [1]

\section{Die verschiedenen Umsetzungen} an den schweizerischen Universitäten

Die Vorreiterrolle bei der Einführung des Bologna-Systems im Medizinstudium übernahmen in der Schweiz die Universitäten Basel und Genf. Der Anfang wurde mit den Studenten gemacht, die sich im Jahr 2006/07 erstmals immatrikulierten, wobei Genf rückwirkend auch noch die vorangegangenen Studiengänge reformierte. Die Umsetzung erfolgte an allen Universitäten der Schweiz in ähnlicher Form. Die sechs Jahre Medizinstudium wurden in drei Jahre Bachelor- und drei Jahre Masterstudium gegliedert, und für jeden dieser Abschnitte können nach dem neu eingeführten Punktesystem 180 ECTS- Credits* angerechnet werden.

Änderungen gab es auch bezüglich der Doktorarbeit, welche man bis anhin noch während des Studiums (meist im dritten und/oder vierten Jahr) verfas- sen konnte. Nun wird während des Masterstudienganges die Masterarbeit geschrieben, welche sowohl für den Studienabschluss als auch den Beginn einer Doktorarbeit Bedingung ist. In Basel darf bereits während des Studiums an der Doktorarbeit geschrieben werden, wohingegen in Zürich zuerst Masterarbeit und Staatsexamen erfolgreich beendet sein müssen. In Bern und auch in Basel wird Wert darauf gelegt, dass die Doktorarbeit auf der Masterarbeit aufbaut.

Die Reform bezieht sich aber nicht nur auf das Ende des Studiums oder die zu schreibenden Arbeiten, sondern auf die ganze Struktur des Lehrplanes. Diese

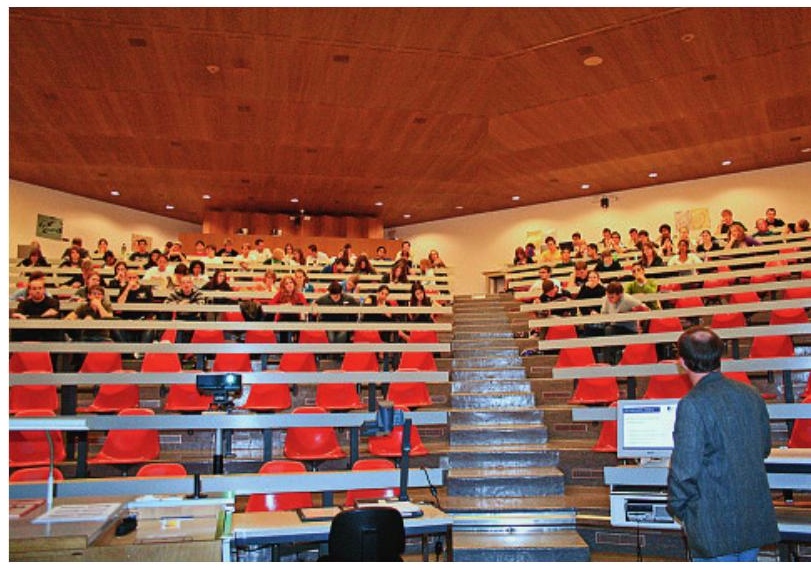

Pathologie-Hörsaal an der Universität Basel: Auch im Bologna-Zeitalter sind die vordersten Reihen bei den Studierenden nicht sonderlich beliebt ..

Photo: Chantal Rast 
beinhaltet, dass neu nicht nur Anatomie und Physiologie in den ersten Jahren gelehrt, sondern dass bereits von Anfang an Bezüge zur Pathophysiologie und Klinik geschaffen werden. Dies bereitete insbesondere den Studierenden aus Fribourg Schwierigkeiten, welche im Herbst 2008 für den dritten Jahreskurs an eine andere Universität mit Bologna-System wechseln mussten.

\section{Probleme der praktischen Umsetzung}

Laura Krüger**, die in diesem Jahr (nach zwei Jahren Grundstudium) von Fribourg nach Basel wechselte, bekam diese Änderung deutlich zu spüren, da sie im alten System begonnen hatte und nun im neuen weiterstudieren soll. Sie klagt über viele Wiederholungen und gleichzeitiges Fehlen von Wissen über Pathologien, welche mit dem Bologna-System in Basel bereits seit dem ersten Studienjahr vermittelt wurden. Doch sie ist nicht die Einzige, die unglücklich ist über das neue Medizinstudium. Auch die Studierenden, welche seit dem ersten Jahreskurs nach dem Bologna-System unterrichtet werden, stellten Unvereinbarkeiten im Lehrplan fest. Beispielsweise berichtet Stefan Hauser**, dass Allergien vor dem Lernen des Immunsystems besprochen sowie die Untersuchungsmethoden eines Auges erklärt wurden, bevor dessen Anatomie den Studenten bekannt war.

\section{Beurteilung des Systems durch die Studenten}

Fünf Studenten des dritten Jahreskurses aus Basel haben für diesen Artikel eine Ad-hoc-Umfrage über die Zufriedenheit der Medizinstudierenden mit der

\section{Grafik 1}

Das Bologna-System stösst bei den Medizinstudierenden in Zürich und Bern (bisher) auf wenig Anklang.

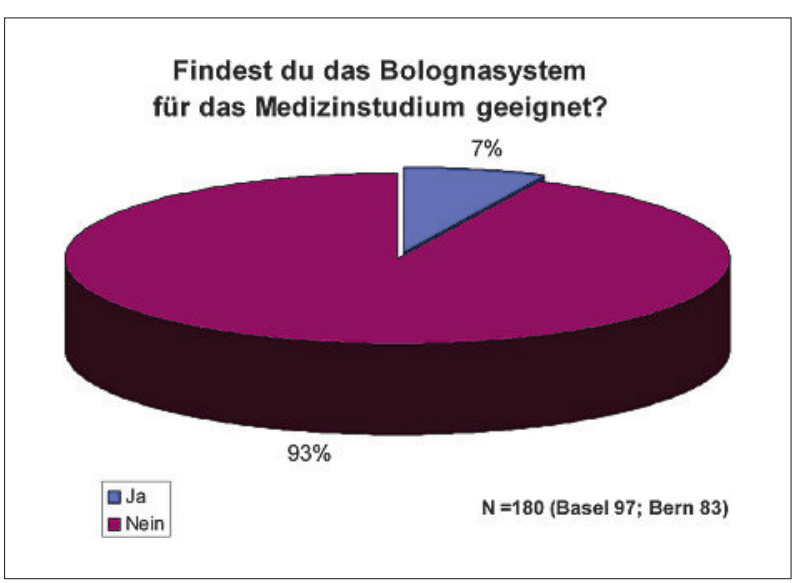

Bologna-Reform durchgeführt. Es wurden rund $280 \mathrm{Me}-$ dizinstudentinnen und -studenten der Universitäten Bern und Basel per E-Mail angeschrieben. Im Zentrum stand die Frage, ob sich die Studenten durch das Bologna-System im Vergleich zur Situation vor der Reform benachteiligt fühlten und ob sie das Bologna-System generell als geeignet für das Medizinstudium einstufen. Die 180 Studierenden, die sich an der Umfrage beteiligt haben, sind sich universitätsübergreifend weitgehend einig: Auffallend ist, wie viele sich gegen das Bologna-System aussprechen. Bei Dreiviertel der Studierenden stösst das neue System auf Ablehnung, und neun von zehn Studenten sind der Meinung, dass das Bologna-System für das Medizinstudium ungeeignet sei. Auch wenn es sich nicht um eine wissenschaftlich fundierte, repräsentative Umfrage handelt, vermittelt sie zumindest ein Stimmungsbild, das darauf hindeutet, dass «Bologna» bei den Medizinstudierenden noch nicht wirklich angekommen ist.

\section{Die Reform des Medizinstudiums*}

Gemäss dem Entscheid des Plenums der CRUS vom 28. Oktober 2005 sieht das neue Modell für das Studium der Humanmedizin ein Bachelorund ein Masterstudium von je 180 ECTS-Credits vor (je drei Jahre bei Vollzeitstudium). Auf Masterstufe sind 60 Credits (ein Jahr Vollzeitstudium) für die klinische Ausbildung vorgesehen. Der Studiengang wird mit der eidg. Prüfung abgeschlossen und bei Erfolg das Eidgenössische Arztdiplom verliehen.

\section{Einführung der neuen Studiengänge}

in der Humanmedizin

Die neuen Bacherlorstudiengänge wurden in der Medizin 2006/07 an den Universitäten Genf, Basel und Freiburg eingeführt. 2007/08 wurde das neue Studiensystem auch in den anderen Universitäten eingeführt. In den Universitäten Genf, Lausanne und Bern fand die Einführung der neuen Studiengänge rückwirkend statt, d.h. Studierende im zweiten Jahr der Universitäten Genf und Bern, respektive im zweiten und dritten Jahr der Universität Lausanne wurden in einen Bachelorstudiengang überführt.

* Ausschnitt (inkl. Titel) aus dem Schlussbericht 2004-07 der CRUS (Rektorenkonferenz der Schweizer Universitäten), Kap. 3.3.2, S 110, zur Reform des Medizinstudiums. Die Vollversion ist im Internet unter www.crus.ch/dms.php?id=6725 einsehbar. 